\title{
Movable $\left(n_{4}\right)$ Configurations
}

\author{
Leah Wrenn Berman
}

Submitted: Jul 17, 2006; Accepted: Nov 8, 2006; Published: Nov 17, 2006

Mathematics Subject Classification: 51A20, 52C35

\begin{abstract}
An $\left(n_{k}\right)$ configuration is a collection of points and straight lines, usually in the Euclidean plane, so that each point lies on $k$ lines and each line passes through $k$ points; such a configuration will be called symmetric if it possesses non-trivial geometric symmetry. Although examples of symmetric $\left(n_{3}\right)$ configurations with continuous parameters are known, to this point, all known connected infinite families of $\left(n_{4}\right)$ configurations with non-trivial geometric symmetry had the property that each set of discrete parameters describing the configuration corresponded to a single $\left(n_{4}\right)$ configuration. This paper presents several new classes of highly symmetric $\left(n_{4}\right)$ configurations which have at least one continuous parameter; that is, the configurations are movable.
\end{abstract}

\section{Introduction}

A geometric $\left(p_{q}, n_{k}\right)$ configuration is a collection of points and straight lines, usually in the Euclidean plane, so that every point lies on $q$ lines and every line passes through $k$ points. By counting incidences (the number of point-line incidences must be equal to the number of line-point incidences), if $p=n$ then $q=k$. Usually, an $\left(n_{k}, n_{k}\right)$ configuration is abbreviated as $\left(n_{k}\right)$. Although $\left(n_{3}\right)$ configurations have been studied since the late $1800 \mathrm{~s},\left(n_{4}\right)$ configurations have been studied for a much shorter time, initially in a series of papers by Branko Grünbaum $[10,11,13]$. Recently, there has been a flurry of activity concerning various kinds of geometric $\left(n_{4}\right)$ configurations (e.g., $[2,3,5,6,7,8]$ ).

There exist connected symmetric $\left(n_{3}\right)$ configurations (for example, astral $\left(n_{3}\right)$ configurations with dihedral symmetry) describable by a set of discrete and continuous parameters $[9,12]$. However, all of the connected $\left(n_{4}\right)$ configurations with 
non-trivial rotational symmetry described previously in the literature (see, for example, $[5,6,10])$ have the property that for a single set of discrete parameters, there is only a single configuration corresponding to those parameters. This paper presents a large family of $\left(n_{4}\right)$ configurations with non-trivial rotational symmetry - they all have $m$-fold rotational symmetry for some $m \geq 8$-where a single set of discrete parameters corresponds to uncountably many $\left(n_{4}\right)$ configurations. That is, the configurations are movable, meaning that they admit a continuous family of realizations fixing four points in general position but moving at least one other point.

It should be noted that it is straightforward to construct highly non-symmetric $\left(n_{k}\right)$ configurations - that is, configurations with no non-trivial geometric symmetrywith a continuous parameter by taking $k$ copies of an $\left(n_{k}\right)$ configuration, each with the same single line removed, translating them in a direction different from those determined by any line of the configuration, and then connecting the points which lie on the removed line with $k$ new lines. Figure 1 shows a $\left(96_{4}\right)$ configuration formed by translating four copies of a $\left(24_{4}\right)$ configuration with a single line deleted from each copy and adding four new lines connecting the necessary points. Parts of the resulting $\left(96_{4}\right)$ configuration corresponding to the original modified $\left(24_{4}\right)$ configurations may be moved (translated) independently.

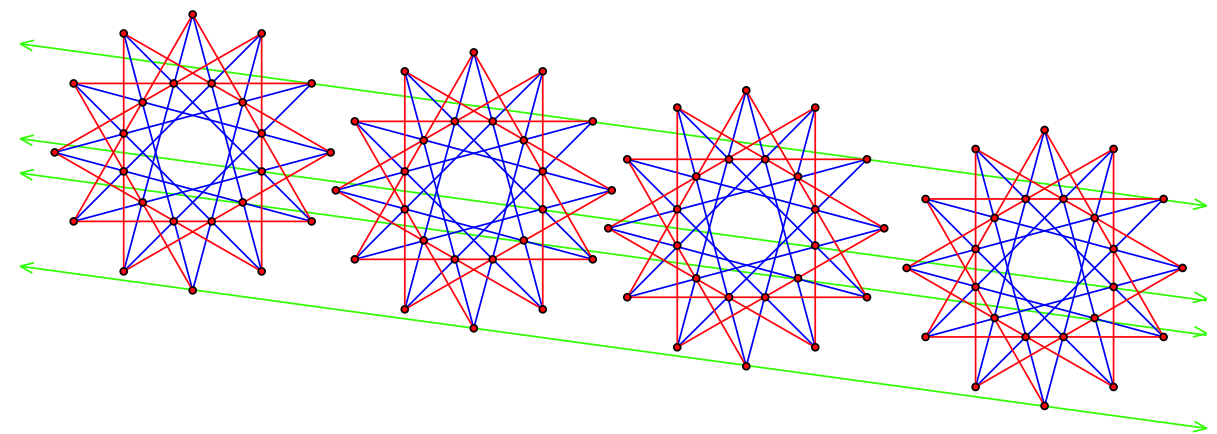

Figure 1: A non-symmetric movable $\left(96_{4}\right)$ configuration, formed by translating four modified copies (each missing a single line) of a $\left(24_{4}\right)$ configuration (with red and blue lines) and appropriately connecting the four copies with four parallel (green) lines. The copies of the configuration may be moved back and forth along the green lines. 


\section{$1.1 \quad$ Preliminary definitions}

Label the vertices of a regular convex $m$-gon consecutively as $w_{0}, \ldots, w_{m-1}$. A diagonal of the $m$-gon is of span $c$ if it connects vertices $w_{i}$ and $w_{i+c}$, where indices are taken modulo $m$. Given a regular polygon and a diagonal of span $c$, label the intersection points of the diagonal with other span $c$ diagonals as $c_{1}, c_{2}, \ldots, c_{\left\lfloor\frac{m}{2}\right\rfloor}$, counted from the midpoint of the diagonal and travelling in one direction (usually, to the left). Following Grünbaum [8], any point on a span $s_{i}$ line which is the $t_{i}$-th intersection (that is, the intersection point with label $s_{i t_{i}}$ ) is given the label $\left[\left[s_{i}, t_{i}\right]\right]$. For an example of this labelling, see Figure 2.

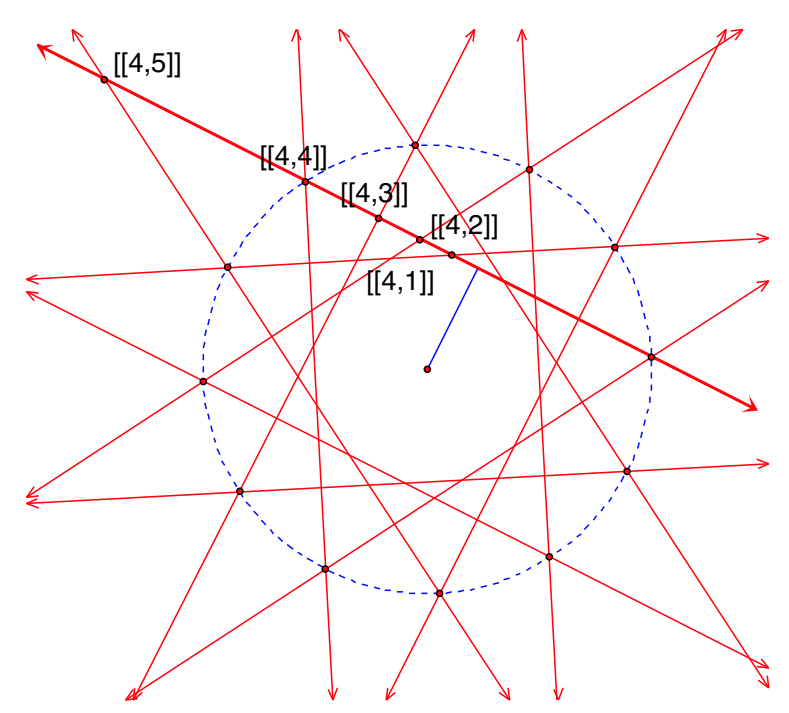

Figure 2: An example of the notation $\left[\left[s_{i}, t_{i}\right]\right]$. Here, $m=12$ and $s_{i}=4$.

\section{Celestial configurations}

All of the movable configurations which will be constructed later in the paper are based on a class of $\left(n_{4}\right)$ configurations, originally developed by Branko Grünbaum [12] and further studied by Marko Boben and Tomaž Pisanski [6] and Grünbaum [8], which have the property that every point has precisely two lines from each of two symmetry classes passing through them, and if there are $m$ points in a symmetry class, then the configuration has the dihedral symmetries of an $m$-gon. These are such a useful class of configurations that having a name to call them would 
be helpful; I propose calling them celestial configurations. To date, celestial configurations were just discussed by referring to their symbol; in [8] Grünbaum uses the symbol $m \#\left(s_{1}, t_{1}, s_{2}, t_{2}, \ldots, s_{h}, t_{h}\right)$, while in [6], the symbol $\mathcal{C}_{4}\left(m,\left(s_{1}, s_{2}, \ldots, s_{h}\right)\right.$, $\left.\left(t_{1}, t_{2}, \ldots t_{h}\right), t\right)$ corresponds to the same configuration. In this paper I am using a modification of the symbol from [8], $m \#\left(s_{1}, t_{1} ; s_{2}, t_{2} ; \ldots ; s_{h}, t_{h}\right)$, to refer to a celestial configuration. Note that a celestial configuration $m \#\left(s_{1}, t_{1} ; s_{2}, t_{2} ; \ldots ; s_{h}, t_{h}\right)$ is an $\left(m h_{4}\right)$ configuration. The earliest drawing of a celestial configuration appeared in a paper by Grünbaum and Rigby [13], where they presented a (214) configuration; other early drawings of celestial configurations appeared as examples in a paper by Marušič and Pisanksi [14].

The following description of celestial configurations is closely based on that given in [8], although it differs slightly in some choices of labelling and point of view. For the sequence $\left(s_{1}, t_{1} ; \ldots ; s_{h}, t_{h}\right)$ to be valid, no two consecutive elements can be equal (and $s_{1} \neq t_{h}$ also).

To construct a celestial configuration $m \#\left(s_{1}, t_{1} ; s_{2}, t_{2} ; \ldots ; s_{h}, t_{h}\right)$ do the following:

1. Begin with $m$ points forming the vertices of a regular $m$-gon; these vertices will be labelled $v_{0,0}, v_{0,1} \ldots, v_{0, m-1}$. Collectively, these vertices will be referred to as $v_{0}$.

2. Draw in lines $L_{0,1}, L_{0,2}, \ldots, L_{0, m-1}$ of span $s_{1}$ connecting these points, so that $L_{0, j}$ connects points $v_{0, j}$ and $v_{0, j+s_{1}}$. These lines will be known collectively as $L_{0}$.

3. Choose the $t_{1}$-st intersection of the span $s_{1}$ lines, counting from the center and moving to the left. Label these vertices as $v_{1,0}, v_{1,1}, \ldots, v_{1, m-1}$, collectively known as $v_{1}$, where $v_{1,0}$ is the $t_{1}$-st intersection point on line $L_{0,0}$. Note that each of the points $v_{1}$ has symbol $\left[\left[s_{1}, t_{1}\right]\right]$.

4. The points $v_{1}$ form the vertices of a regular $m$-gon; using these vertices, draw in diagonals of span $s_{2}$ and label them as $L_{1,0}, L_{1,1}, \ldots, L_{1, m-1}$ as above (and collectively as $\left.L_{1}\right)$.

5. Choose points on these lines which are the $t_{2}$-nd intersection of the span $s_{2}$ lines, counting from the center (with label $\left[\left[s_{2}, t_{2}\right]\right]$ ) and label them as $v_{2,0}, v_{2,1}, \ldots, v_{2, m-1}$, or collectively as $v_{2}$, with $v_{2,0}$ the $t_{2}$-nd intersection point on line $L_{1,0}$.

6. Continue in this fashion until lines of span $s_{h}$ are constructed using points with label $v_{h}$; if the symbol correctly identifies an $\left(n_{4}\right)$ configuration, then the points $v_{h}$ with label $\left[\left[s_{h}, t_{h}\right]\right]$ will coincide with the original $m$ points labelled $v_{0}$. 
It is important to note that several symbols may correspond to the same geometric configuration, although the labelling of the points and lines depends on the precise choice of symbol. For an example of this, see Figure 3. In particular, the points labelled $v_{0}$ need not be the outermost ring of points, as in Figure 3(b).

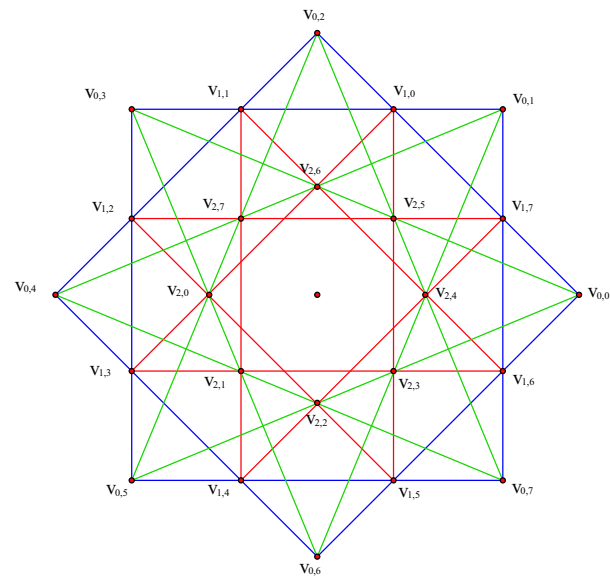

(a)

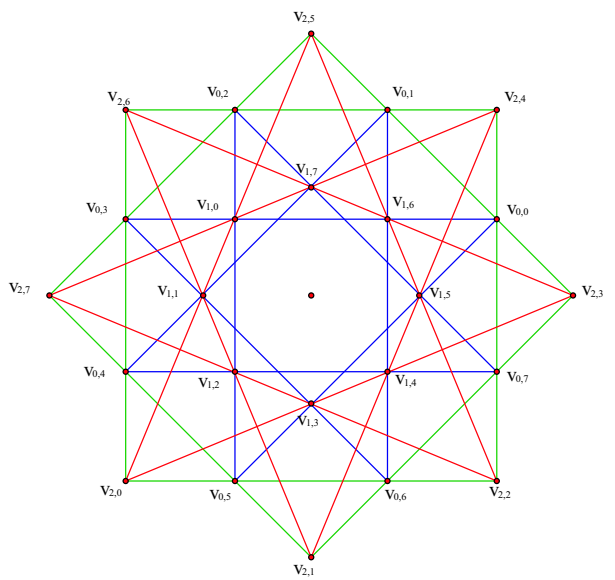

(b)

Figure 3: Two symbols corresponding to the same celestial configuration, with labels. (a) $8 \#(2,1 ; 3,2 ; 1,3)$; (b) $8 \#(3,2 ; 1,3 ; 2,1)$. In each configuration, lines $L_{0}$ are blue, $L_{1}$ are red, and $L_{2}$ are green.

In [8] and [6] it is shown that given a symbol $m \#\left(s_{1}, t_{1} ; s_{2}, t_{2} ; \ldots ; s_{h}, t_{h}\right)$, reversing the sequence or cyclically permuting the sequence $\left(s_{1}, t_{1} ; s_{2}, t_{2} ; \ldots ; s_{h}, t_{h}\right)$ using permutations that advance the sequence an even number of places leads to an equivalent configuration, while advancing an odd number of places yields a polar configuration. Grünbaum listed two conditions, labelled $\left(^{*}\right)$ and $\left({ }^{*}\right)$ below, that must hold in order for a celestial configuration to exist (taken from [8]):

$$
\begin{array}{r}
s_{1}+t_{1}+s_{2}+t_{2} \cdots+s_{h}+t_{h} \text { is even } \\
\frac{\cos \left(\frac{\pi s_{1}}{m}\right)}{\cos \left(\frac{\pi t_{1}}{m}\right)} \cdot \frac{\cos \left(\frac{\pi s_{2}}{m}\right)}{\cos \left(\frac{\pi t_{2}}{m}\right)} \cdot \cdots \cdot \frac{\cos \left(\frac{\pi s_{h}}{m}\right)}{\cos \left(\frac{\pi t_{h}}{m}\right)}=1
\end{array}
$$

In addition, for the configuration to be connected, if $m, s_{1}, s_{2}, \ldots s_{h}, t_{1}, t_{2}, \ldots t_{h}$ have a common factor $f$, then the symbol

$$
\frac{m}{f} \#\left(\frac{s_{1}}{f}, \frac{t_{1}}{f} ; \ldots ; \frac{s_{h}}{f}, \frac{t_{h}}{f}\right) \text { must not satisfy conditions }(*) \text { and }(* *)
$$


(or else the original configuration consists of $f$ concentric copies of the smaller configuration $\frac{m}{f} \#\left(\frac{s_{1}}{f}, \frac{t_{1}}{f} ; \ldots ; \frac{s_{h}}{f}, \frac{t_{h}}{f}\right)$, rotated so the copies are evenly spaced).

Of particular utility are the trivial configurations, where the unordered set of $s_{j}$ 's is the same as the unordered set of $t_{j}$ 's, so that conditions $\left(^{*}\right)$ and $\left({ }^{*}\right)$ are automatically satisfied; the configuration in Figure 3 is a trivial configuration.

\section{$3 \quad$ Removing half of a symmetry class}

The movable $\left(n_{4}\right)$ configurations will be constructed by "nesting" modified celestial configurations so that two sets of vertices of one configuration lie on the two sets of lines of a second configuration. However, simply nesting two configurations in this fashion would lead to sets of points with five lines passing through them and sets of lines with five points on them. In order to end up with an $\left(n_{4}\right)$ configuration, we must delete half the points in one symmetry class of one configuration and half the lines in one symmetry class in the second configuration.

Note that to be able to remove half the objects in a symmetry class, the number of objects, namely $m$, must be even!

It is helpful to analyze carefully the notation for celestial configurations presented in the previous section. Consider a configuration $m \#\left(s_{1}, t_{1} ; s_{2}, t_{2} ; \ldots ; s_{h}, t_{h}\right)$. It has points $v_{0}=\left[\left[s_{h}, t_{h}\right]\right], v_{1}=\left[\left[s_{1}, t_{1}\right]\right], \ldots, v_{h-1}=\left[\left[s_{h-1}, t_{h-1}\right]\right]$. It also has lines $L_{0}$, $L_{1}, \ldots, L_{h-1}$, where each class of lines $L_{i}$ contains points with label $v_{i}$ and $v_{i+1}$, so each point $v_{i}$ has lines $L_{i}$ and $L_{i-1}$ passing through it, of spans as presented in Table 1.

\begin{tabular}{c||c|c||c|c} 
point & line & span & line & span \\
$v_{0}$ & $L_{0}$ & $s_{1}$ & $L_{h-1}$ & $t_{h}$ \\
$v_{1}$ & $L_{1}$ & $s_{2}$ & $L_{0}$ & $t_{1}$ \\
$\vdots$ & $\vdots$ & $\vdots$ & $\vdots$ & $\vdots$ \\
$v_{i-1}$ & $L_{i-1}$ & $s_{i}$ & $L_{i-2}$ & $t_{i-1}$ \\
$v_{i}$ & $L_{i}$ & $s_{i+1}$ & $L_{i-1}$ & $t_{i}$ \\
$\vdots$ & $\vdots$ & $\vdots$ & $\vdots$ & $\vdots$ \\
$v_{h-1}$ & $L_{h-1}$ & $s_{0}$ & $L_{h-2}$ & $t_{h-1}$
\end{tabular}

Table 1: Spans of lines, with their labels, passing through points $v_{i}$, from the point of view of that class of points.

When considering how to delete half the points or lines in a particular symmetry 
class, we will look at the interaction of points and lines carefully, from the point of view of different classes of points and lines.

The statement "half the lines in a symmetry class may be removed" means that if the lines in the symmetry class are labelled $L_{i, 0}, L_{i, 1}, \ldots, L_{i, m-1}$, where each line has four points lying on it, then removing the every other line in the symmetry class - for example, removing lines $L_{i, 0}, L_{i, 2}, \ldots$ with even index - leaves one line of the symmetry class passing through each point in the symmetry class $v_{i+1}$, rather than some points having two lines of the symmetry class incident and others having none.

Lemma 1. Half of the lines of span $s_{i}$ passing through the points labelled $\left[\left[s_{i}, t_{i}\right]\right]$ may be removed precisely when $s_{i}$ and $t_{i}$ are both odd.

Proof. Following the notation in the section on celestial configurations, label the vertices $\left[\left[s_{i-1}, t_{i-1}\right]\right]$ as $v_{i-1,0}, v_{i-1,1}, \ldots, v_{i-1, m-1}$, and label the lines of span $i$ as $L_{i, 0}, L_{i, 1}, \ldots, L_{i, m-1}$; note that $L_{i, j}$ contains points $v_{i-1, j}$ and $v_{i-1, j-s_{i}}$. Label the vertices $\left[\left[s_{i}, t_{i}\right]\right]$ as $v_{i, 0}, v_{i, 1}, \ldots, v_{i, m-1}$, where $v_{i, 0}$ is the $t_{i}$-th intersection of line $L_{i, 0}$ with other span $s_{i}$ lines. Then line $L_{i, j}$ also contains points $v_{i, j}$ and $v_{i, j-t_{i}}$; that is, they are span $t_{i}$ lines with respect to the $v_{i, j}$.

Suppose that lines $L_{i, 0}, L_{i, 2}, \ldots$ are removed. Point $v_{i-1, j}$ contains lines $L_{i, j}$ and $L_{i, j-s_{i}}$. If $s_{i}$ is even, then whenever $j$ is even, both lines $L_{i, j}$ and $L_{i, j-s_{i}}$ will be removed from point $v_{i, j}$. On the other hand, if $s_{i}$ is odd, then each $v_{i-1, j}$ will have a single line $L_{i}$ removed. Similarly, considering the lines $L_{i}$ to be lines of span $t_{i}$ passing through the points $v_{i}$, each $v_{i, j}$ will have a single line $L_{i}$ passing through it precisely when $t_{i}$ is odd.

Therefore, if lines $L_{i, 0}, L_{i, 2}, \ldots$ are removed, the points $v_{i-1, j}$ and $v_{i, j}$ will each have a single line $L_{i}$ passing through them when both $s_{i}$ and $t_{i}$ are odd.

A similar argument holds if lines with odd index $L_{i, 1}, L_{i, 3}, \ldots$ are removed.

The statement "half the points in a symmetry class may be removed" means the following: Choose one symmetry class of points $\left[\left[s_{i}, t_{i}\right]\right]$; they have labels $v_{i, 0}, v_{i, 1}$, $\ldots, v_{i, m-1}$. Each point has four lines passing through it, two of span $t_{i}$ and two of span $s_{i+1}$. We wish to remove every other point in a symmetry class - for example, points $v_{i, 0}, v_{i, 2}, \ldots$ with even index - in such a way that each of the lines of span $t_{i-1}$ and span $s_{i}$ have a single point labelled $v_{i, j}$, rather than some lines having two points labelled $v_{i, j}$ and some lines having none.

Lemma 2. Half the points in the symmetry class $\left[\left[s_{i}, t_{i}\right]\right]$ may be removed precisely when $t_{i}$ and $s_{i+1}$ are both odd. 
Proof. Consider the points $\left[\left[s_{i+1}, t_{i+1}\right]\right]$. Label them $v_{i+1,0}, v_{i+1,1}, v_{i+1,2}, \ldots, v_{i+1, m-1}$. The $v_{i+1}$ lie on lines of span $s_{i+1}$, labelled as $L_{i+1}$, where line $L_{i+1, j}$ contains points $v_{i+1, j}$ and $v_{i+1, j-s_{i+1}}$. Now remove the points $v_{i+1, q}$ where $q$ is even. If $s_{i+1}$ is even, then in line $L_{i+1, j}$ if $j$ is even, both points $v_{i+1, j}$ and $v_{i+1, j-s_{i+1}}$ will be removed, while if $j$ is odd, neither point will be removed. On the the other hand, if $s_{i+1}$ is odd, in line $L_{i+1, j}$, exactly one of the points $v_{i+1, j}$ or $v_{i+1, j-s_{i+1}}$ will be removed, since their indices are of different parity. Similarly, in the situation where points $v_{i+1, q}$ where $q$ is odd, are removed, if $s_{i+1}$ is odd, then in line $L_{i+1, j}$, exactly one of the points $v_{i+1, j}$ or $v_{i+1, j-s_{i+1}}$ will be removed, while if $s_{i+1}$ is even, both points or neither point will be removed.

The points $v_{i+1}$ also lie on lines $L_{i}$, as the $t_{i}$-th intersection of span $s_{i}$ lines. From the point of view of the points $v_{i+1}$, the lines $L_{i}$ are span $t_{i}$ lines; that is, the points $v_{i+1}$ are the $s_{i}$-th intersection of the span $t_{i}$ lines. Using this point of view, the previous argument shows that half of these points may be removed from the span $t_{i}$ lines, leaving only one point labelled $v_{i+1}$ on each line, precisely when $t_{i}$ is odd.

The method of constructing movable configurations which will be presented here will begin with one modifed celestial configuration $\mathcal{C}$ which has a symmetry class of points, called $S$, with half its points removed, and a second modified celestial configuration $\mathcal{D}$, with the same value for $m$, which has had half of an appropriate symmetry class of lines removed. These configurations must be chosen so that when an arbitrary point is placed on a line in $\mathcal{C}$ containing points in $S$ and then rotated to form an $m$-gon, and $\mathcal{D}$ is constructed using these points as its starting $m$-gon, one of the symmetry classes of points in $\mathcal{D}$ lies on a class of lines in $\mathcal{C}$.

In Figure 4, diagonals of span 2 and span 3 are shown (in blue and red, respectively), along with an arbitrary point placed on a blue line and rotated around to form another 10-gon, and a set of green lines which are diagonals of span 3 for the second 10-gon. Note that the second intersection point of the green lines with each other lies on the red lines.

Theorem 3. Given a regular $m$-gon with vertices $u_{0}, u_{1}, \ldots, u_{m-1}$ and diagonals of span a and span b, suppose that $w_{0}$ is an arbitrary point on the 0 -th diagonal of span $a$, which joins $u_{0}$ and $u_{a}$ and is denoted by $\left\langle u_{0}, u_{a}\right\rangle$, and the other $w_{i}$ are formed by rotating $w_{0}$ by $\frac{2 \pi i}{m}$. Let $q_{i}$ be the intersection of the line $\left\langle w_{i}, w_{i+b}\right\rangle$ with the span $b$ diagonal $\left\langle u_{i}, u_{i+b}\right\rangle$, and let $q_{i}^{\prime}$ be the intersection of the lines $\left\langle w_{i-a}, w_{i-a+b}\right\rangle$ and $\left\langle u_{i}, u_{i+b}\right\rangle$. Then $q_{i}=q_{i}^{\prime}$.

That is, if you begin with a set of diagonals of span $a$ and span $b$ of an $m$-gon $M$, construct another $m$-gon $N$ whose vertices are the rotated images of a point placed 


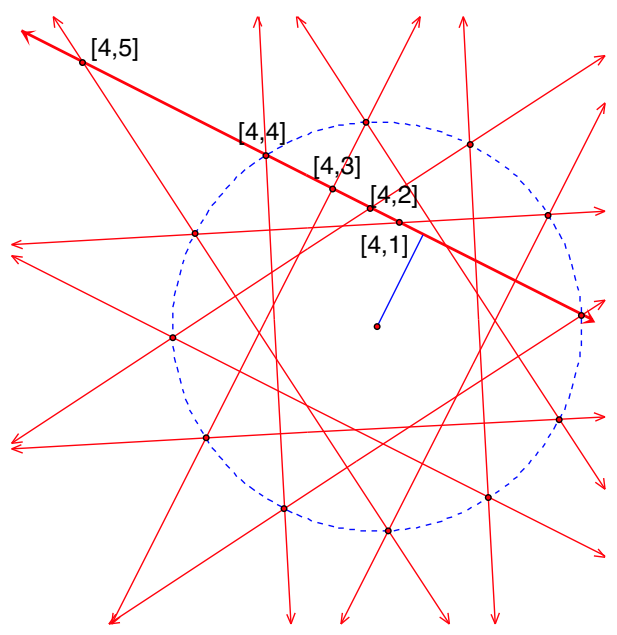

Figure 4: Beginning with a regular $m$-gon with diagonals of span $a$ (blue lines) and $b$ (red lines), constructing a second $m$-gon with diagonals of span $b$ (green lines) whose vertices are the rotated images of a point placed arbitrarily on a diagonals of span $a$ leads to these diagonals of span $b$ intersecting the original diagonals of span $b$. In this figure, $m=10, a=2$ and $b=3$.

arbitrarily on a diagonal of span $a$, and construct diagonals of span $b$ using $N$, then these diagonals intersect the span $b$ diagonals of $M$, and the intersection points are precisely the points labelled $[[b, a]]$ in $N$.

To prove this lemma, I will need two geometric results.

Lemma 4. If quadrilateral $\square Q R S T$ has the property that $\angle R Q S=$ $\angle R T S$, then the points $Q, R, S$, and $T$ are concyclic.

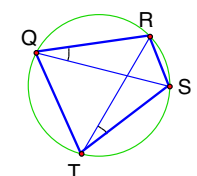

This lemma is a well-known result from Euclidean geometry; see, for example, [1, p. 127]

Lemma 5. Let $M$ and $N$ be two concentric m-gons with center $\mathcal{O}$ and vertices $u_{0}, u_{1}, \ldots, u_{m-1}$ and $w_{0}, w_{1}, \ldots, w_{m-1}$, respectively. Let $\ell_{1}$ be the line $\left\langle u_{0}, u_{s}\right\rangle$, a diagonal of $M$ of span $s$, and let $\ell_{2}$ be the line $\left\langle w_{i}, w_{i+s}\right\rangle$, a diagonal of $N$ of span s. Let $q$ be the intersection of $\ell_{1}$ and $\ell_{2}$. Then the four points $u_{0}, w_{i}, \mathcal{O}$ and $q$ are concyclic.

Proof. See Figure 5(a) for an illustration. Let $M_{u}$ be the midpoint of $u_{0} u_{s}$ and let 
$M_{w}$ be the midpoint of $w_{i} w_{i+s}$. Then

$$
\angle u_{0} \mathcal{O} M_{u}=\frac{\pi s}{m}, \quad \angle w_{i} \mathcal{O} M_{w}=\frac{\pi s}{m}, \quad \angle u_{0} M_{u} \mathcal{O}=\frac{\pi}{2}, \quad \text { and } \quad \angle w_{i} M_{w} \mathcal{O}=\frac{\pi}{2},
$$

since the angle between endpoints of a segment of span $s$ is $\frac{2 \pi s}{m}$ and the segments $M_{u} \mathcal{O}$ and $M_{w} \mathcal{O}$ are perpendicular to the diagonals $\ell_{1}$ and $\ell_{2}$ respectively. It follows that $\angle M_{u} u_{0} \mathcal{O}=\angle M_{w} w_{i} \mathcal{O}$. But $q$ lies on $\ell_{1}$ and $\ell_{2}$, so $\angle q u_{0} \mathcal{O}=\angle q w_{i} \mathcal{O}$. By Lemma $4, q, w_{i}, u_{0}$ and $\mathcal{O}$ are concyclic.

We will apply this result several times to prove Theorem 3; see Figure 5(b).

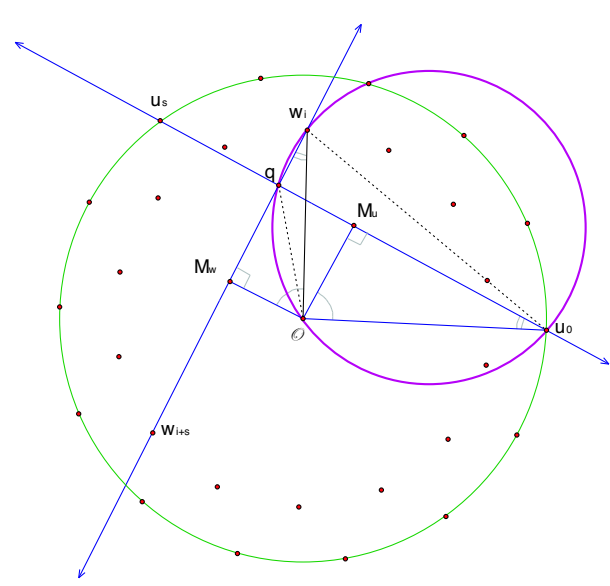

(a)

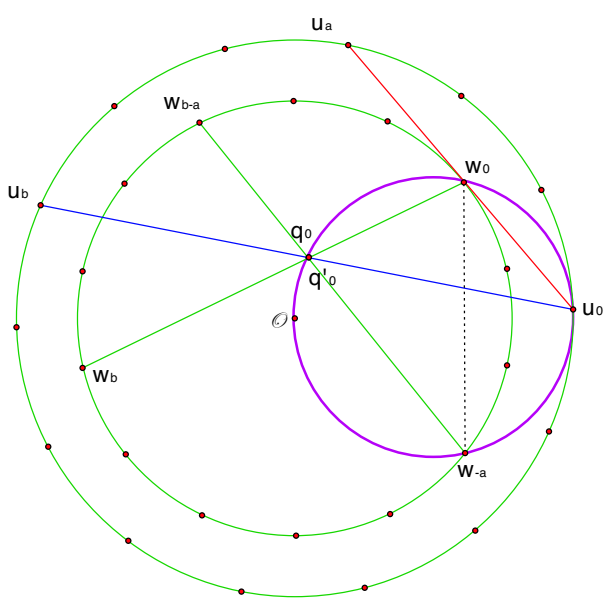

(b)

Figure 5: Illustrations for the proofs of (a) Lemma 5 and (b) Theorem 3.

Proof of Theorem 3. Let $M$ be an $m$-gon with center $\mathcal{O}$, vertices $u_{0}, u_{1}, \ldots u_{m-1}$ and diagonals of span $a$ and span $b$, let $w_{0}$ be an arbitrary point on line $\left\langle u_{0}, u_{a}\right\rangle$, and let the other $w_{i}$ be formed by rotating $w_{0}$ by $\frac{2 \pi i}{m}$. Let $q_{i}$ be the intersection of line $\left\langle w_{i}, w_{i+b}\right\rangle$ with the span $b$ diagonal $\left\langle u_{i}, u_{i+b}\right\rangle$, and let $q_{i}^{\prime}$ be the intersection of the lines $\left\langle w_{i-a}, w_{i-a+b}\right\rangle$ and $\left\langle u_{i}, u_{i+b}\right\rangle$.

By symmetry, it suffices to show that $q_{0}=q_{0}^{\prime}$. Since $q_{0}$ is the intersection of $\left\langle w_{0}, w_{b}\right\rangle$ and $\left\langle u_{0}, u_{b}\right\rangle$, by Lemma $5, q_{0}, w_{0}, u_{0}$ and $\mathcal{O}$ are concyclic. Similarly, $q_{0}^{\prime}$ lies on the same circle as $\mathcal{O}, u_{0}$, and $w_{-a}$, since it lies on the intersection of span $b$ diagonals $\left\langle w_{-a}, w_{-a+b}\right\rangle$ and $\left\langle u_{0}, u_{b}\right\rangle$. Finally, since the lines $\left\langle w_{-a}, w_{0}\right\rangle$ and $\left\langle u_{0}, u_{a}\right\rangle$ are both of span $a$ and intersect at the point $w_{0}$, it follows that the points $w_{0}, w_{-a}, u_{0}$, and $\mathcal{O}$ are concyclic as well, again by Lemma 5. Since a circle is uniquely determined 
by three points, the points $q_{0}, q_{0}^{\prime}, w_{0}, w_{-a}, u_{0}$ and $\mathcal{O}$ all lie on the same circle, $\mathcal{C}$. Since the line $\left\langle u_{0}, u_{b}\right\rangle$ can intersect $\mathcal{C}$ only twice, with one of the intersections at $u_{0}$, and since both $q_{0}$ and $q_{0}^{\prime}$ lie on $\mathcal{C}$ and the line $\left\langle u_{0}, u_{b}\right\rangle$ and are not $u_{0}$, it follows that $q_{0}=q_{0}^{\prime}$.

\section{Movable configurations}

This section will present several interesting classes of movable configurations, along with explicit examples. The basic movable configuration is constructed from two modified celestial configurations (which are no longer configurations, since not all points lie on the same number of lines), one with half the points of a symmetry class of points removed and one with half the lines of a symmetry class of lines removed, so that two of the symmetry classes of points of one modified configuration lie arbitrarily on two symmetry classes of lines of the other modified configuration.

\subsection{The combined symbol}

We will use a combined symbol to represent a potential movable configuration constructed from two modified celestial configurations.

We will call a configuration modifiable if it contains two consecutive odd numbers in its sequence. If the consecutive odd numbers are of the form $t_{i} ; s_{i+1}$ then the configuration is point-modifiable, meaning that one half of the corresponding symmetry class of points $v_{i+1}$ with label $\left[\left[s_{i}, t_{i}\right]\right]$ (either with even index or with odd index) may be removed, by Lemma 2 . If the consecutive odd numbers are of the form $s_{i}, t_{i}$ then we shall call the configuration line-modifiable, meaning that half of the corresponding symmetry class of lines, $L_{i-1}$, may be removed (again, using either even or odd index), by Lemma 1.

To indicate that half the points $\left[\left[s_{i}, t_{i}\right]\right]$ of a point-modifiable configuration have been removed, we place an asterisk in front of $t_{i}$; for example, the modified configuration $8 \#(2, * 1 ; 3,2 ; 1,3)$ shown in Figure $6($ a) refers to a configuration with half of the points labelled [[2,1]] removed. Similarly, an asterisk in front of $s_{i}$ in a line-modifiable configuration indicates that half the lines with label $L_{i-1}$ have been removed; in $8 \#(* 3,1 ; 2,3 ; 1,2)$, shown in Figure $6(\mathrm{~b})$, half the lines $L_{0}$ have been removed. In general, we permute the sequences as necessary so that the asterisk corresponding to the removed points is in the second position in the sequence, and the asterisk indicating the removed lines is in the first position in the sequence, although in more complicated constructions (see Section 5) this is not always possible.

THE ELECTRONIC JOURNAL OF COMBINATORICS 13 (2006), \#R104 


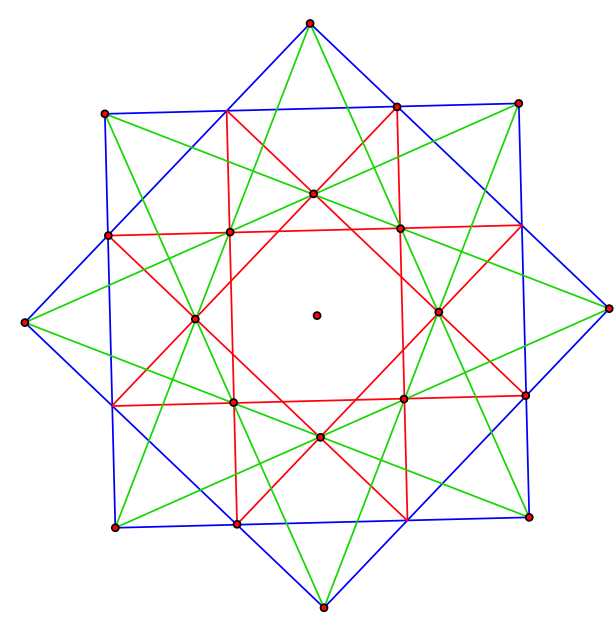

(a)

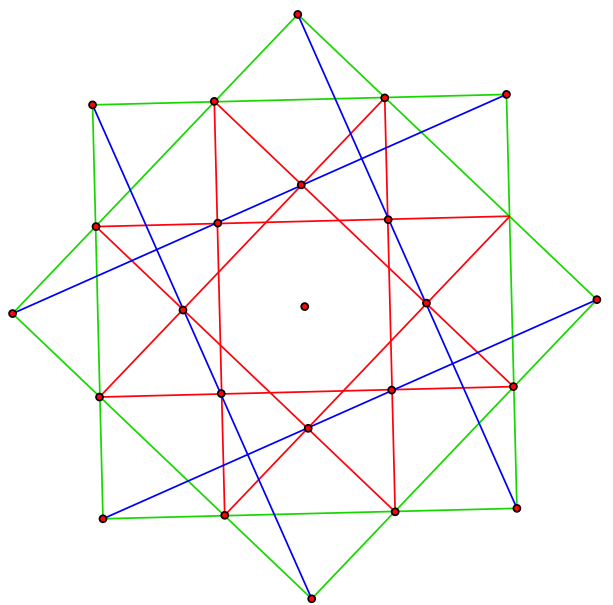

(b)

Figure 6: Modified configurations. (a) The modified configuration $8 \#(2, * 1 ; 3,2 ; 1,3)$, with half the points labelled $[[2,1]]$ removed; (b) the modified configuration $8 \#(* 3,1 ; 2,3 ; 1,2)$, with half the (blue) lines $L_{0}$ removed.

Given a point-modifiable configuration $\mathcal{C}=m \#\left(s_{1}, * t_{1} ; \ldots ; s_{h}, t_{h}\right)$, with half the points labelled $\left[\left[s_{1}, t_{1}\right]\right]$ removed, and a line-modifiable configuration $\mathcal{D}=m \#\left(* q_{1}, r_{1} ; \ldots ; q_{k}, r_{k}\right)$, with half the lines $L_{0}^{\prime}$ removed, we can write down the combined symbol

$$
m \#\left(s_{1}, * t_{1} ; \ldots ; s_{h}, t_{h}\right) \$\left(* q_{1}, r_{1} ; \ldots ; q_{k}, r_{k}\right),
$$

which represents a potential configuration that is constructed from the two modified configurations as follows:

1. Construct $\mathcal{C}=m \#\left(s_{1}, * t_{1} ; \ldots ; s_{h}, t_{h}\right)$

2. Place an arbitrary point $v_{0,0}^{\prime}$ on line $L_{0,0}$ of $\mathcal{C}$, and create $m-1$ copies by rotating it by $\frac{2 i \pi}{m}$, where $i=1, \ldots, m-1$.

3. Label these points as $v_{0, i}^{\prime}$, and use them as the starting $m$-gon to create the modified configuration $\mathcal{D}=m \#\left(* q_{1}, r_{1} ; \ldots ; q_{k}, r_{k}\right)$.

We call a combined symbol

$$
m \#\left(s_{1}, * t_{1} ; \ldots ; s_{h}, t_{h}\right) \$\left(* q_{1}, r_{1} ; \ldots ; q_{h}, r_{h}\right)
$$


admissible if the points $v_{1}^{\prime}$ of the modified configuration $m \#\left(* q_{1}, r_{1} ; \ldots ; q_{k}, r_{k}\right)$ lie on the lines $L_{1}$ of the modified configuration $m \#\left(s_{1}, * t_{1} ; \ldots ; s_{i}, t_{i} ; \ldots ; s_{h}, t_{h}\right)$. If the combined symbol is admissible, we will say the two component configurations are compatible.

Theorem 6. Admissible combined symbols correspond to movable $\left(n_{4}\right)$ configurations.

Proof. Consider the collection of points and lines corresponding to an admissible combined symbol

$$
m \#\left(s_{1}, * t_{1} ; \ldots ; s_{h}, t_{h}\right) \$\left(* q_{1}, r_{1} ; \ldots ; q_{k}, r_{k}\right)
$$

that is formed by following steps $1-3$ above. To show this collection of points and lines is an $\left(n_{4}\right)$ configuration, we need to show that every point has four lines passing through it and every line has four points lying on it. The modified configuration $\mathcal{C}=m \#\left(s_{1}, * t_{1} ; \ldots ; s_{h}, t_{h}\right)$ has had half of the points $\left[\left[s_{1}, t_{1}\right]\right]$ that lie on lines $L_{0}$ and $L_{1}$ removed. In the construction of the modified configuration $\mathcal{D}=m \#\left(* q_{1}, r_{1} ; \ldots ; q_{k}, r_{k}\right)$, new points $v_{0}^{\prime}$ were placed on the lines $L_{0}$, so now each line $L_{0}$ contains two points with label $v_{0}$, one point with label $v_{1}$, and one point with label $v_{0}^{\prime}$. All the other lines $L_{i}$ of $\mathcal{C}$, with the exception of the lines $L_{1}$, have not been modified and so still contain four points, and all the points that lie on $\mathcal{C}$, with the exception of the new points labelled $v_{0}^{\prime}$, contain four lines.

Since $\mathcal{D}$ has had half of its lines labelled $L_{0}^{\prime}$ removed, when it is constructed using the $v_{0}^{\prime}$, each $v_{0}^{\prime}$ will have passing through it one line labelled $L_{0}$ (from $\mathcal{C}$ ), one line labelled $L_{0}^{\prime}$, and two lines labelled $L_{1}^{\prime}($ from $\mathcal{D})$. All the other points in $\mathcal{D}$ lie on two lines from $\mathcal{D}$, with the exception of the points labelled $v_{1}^{\prime}$. Since the points $v_{1}^{\prime}$ lie on the lines $L_{0}^{\prime}$, half of which were removed, they only have three lines from $\mathcal{D}$ passing through them. However, if the points labelled $v_{1}^{\prime}$ also lie on the lines labelled $L_{1}$ from $\mathcal{C}$, because the combined symbol was assumed to be admissible, then every point will lie on four lines and every line will pass through four points, as is necessary for the $\left(n_{4}\right)$ configuration to exist.

Finally, the points $v_{0}^{\prime}$ may move at will along the lines $L_{0}$ while the modified configuration $\mathcal{C}$ is stationary, so the configuration corresponding to the combined symbol has a single degree of freedom.

Note that the configuration corresponding to an admissible symbol

$$
m \#\left(s_{1}, * t_{1} ; \ldots ; s_{h}, t_{h}\right) \$\left(* q_{1}, r_{1} ; \ldots ; q_{k}, r_{k}\right),
$$

is a $\left(2 m(h+k)_{4}\right)$ configuration. 
Figures 7 and 8 show two movable configurations with admissible combined symbols. The next section will present some constraints on the sequences of the underlying celestial configurations that correspond to admissible combined symbols. The configuration shown in Figure 7 has combined symbol

$$
8 \#(2, * 1 ; 3,2 ; 1,3) \$(* 3,1 ; 2,3 ; 1,2) \text {. }
$$

The yellow point indicated in this figure by $v_{0}^{\prime}$ may move freely along the thick blue line. In this figure, as in all figures in the rest of the paper, line segments, rather than lines, are used in order to reduce clutter in the diagram, but the intention is to indicate that a point placed on a line may move anywhere along the entire line (even beyond the end of the pictured segment). It is critical in the subsequent analysis that the configurations are thought of as being composed of lines rather than segments. Note that for a discrete number of positions, as the point $v_{0}^{\prime}$ moves along a line $L_{1}$, unwanted coincidences will occur, yielding for that position a representation of the underlying combinatorial configuration rather than a realization.

Interestingly, it is possible to combine two configurations which have different numbers of symmetry classes! Figure 8 shows a movable configuration constructed from $12 \#(4,3 ; 1,2 ; 3,4 ; 2,1)$, which has four symmetry classes of points and lines, and $12 \#(1,3 ; 5,1 ; 3,5)$, which has only three symmetry classes of points and lines.

\subsection{A class of movable configurations}

A large class of movable configurations may be constructed by combining two celestial configurations $m \#\left(s_{1}, t_{1} ; s_{2}, t_{2} ; \ldots ; s_{h}, t_{h}\right)$ and $m \#\left(s_{2}, t_{1} ; q_{2}, r_{2} ; \ldots ; q_{k}, r_{k}\right)$, where $t_{1}$ and $s_{2}$ are both odd.

Theorem 7. If $t_{1}$ and $s_{2}$ are both odd, then the combined symbol

$$
m \#\left(s_{1}, * t_{1} ; s_{2}, t_{2} ; \ldots, s_{h}, t_{h}\right) \$\left(* s_{2}, t_{1} ; q_{2}, r_{2}, \ldots ; q_{k}, r_{k}\right)
$$

is admissible.

Proof. Let $\mathcal{C}=m \#\left(s_{1}, t_{1} ; s_{2}, t_{2} ; \ldots, s_{h}, t_{h}\right)$ and $\mathcal{D}=m \#\left(s_{2}, t_{1} ; q_{2}, r_{2}, \ldots ; q_{k}, r_{k}\right)$. Since $t_{1}$ and $s_{2}$ are both odd, $\mathcal{C}$ is point-modifiable and $\mathcal{D}$ is line-modifiable.

Note that in the construction of the collection of points and lines corresponding to the combined symbol, we place new points $v_{0}^{\prime}$ on the lines $L_{0}$, which are of span $s_{1}$. From the point of view of the points $v_{1}$, the lines $L_{0}$ are of span $t_{1}$; the second class of lines passing through the points $v_{1}$ are $L_{1}$, which are of span $s_{2}$ with respect to the $v_{1}$. 


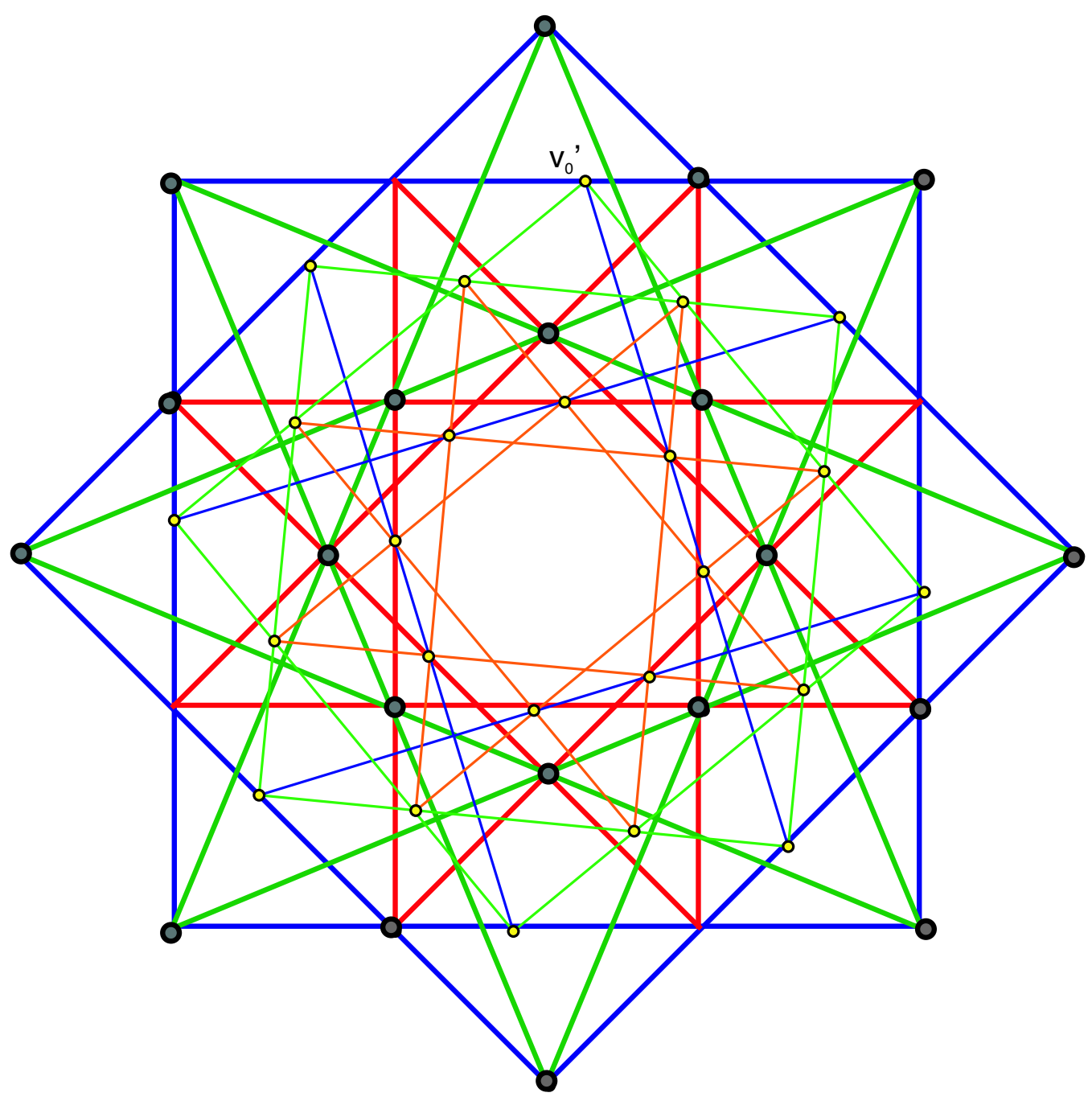

Figure 7: A movable configuration with symbol $8 \#(2, * 1 ; 3,2 ; 1,3) \$(* 3,1 ; 2,3 ; 1,2)$. The modified configuration $8 \#(2, * 1 ; 3,2 ; 1,3)$ is shown with thick lines, and the modified configuration $8 \#(* 3,1 ; 2,3 ; 1,2)$ is shown with thin lines. 


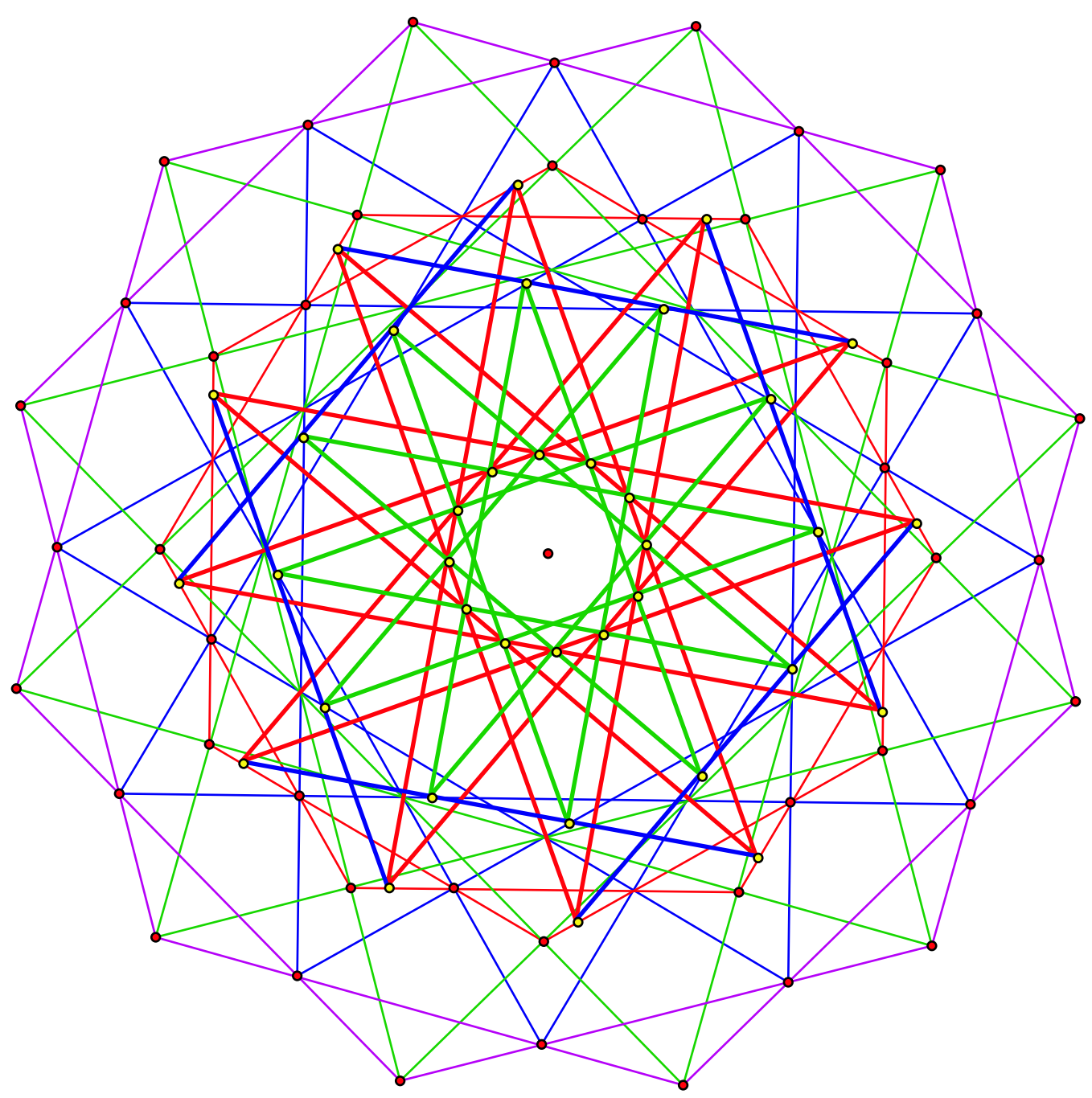

Figure 8: A movable configuration with symbol

$$
12 \#(4,3 ; * 1,2 ; 3,4 ; 2,1) \$(* 1,3 ; 5,1 ; 3,5) \text {. }
$$

The modified configuration $12 \#(4,3 ; * 1,2 ; 3,4 ; 2,1)$ is shown with thin lines, and the modified configuration $12 \#(* 1,3 ; 5,1 ; 3,5)$ is shown with thick lines. 
To show that the combined symbol is admissible, we must show that the modified configuration constructed using the points $v_{0}^{\prime}$ has the symmetry class of points labelled $v_{1}^{\prime}$ lying on the lines $L_{1}$ of the original configuration. The points $v_{0}^{\prime}$ contain lines $L_{0}^{\prime}$, of span $s_{2}$, and in the construction of the second configuration, the $t_{1}$-th intersection points of the lines $L_{0}^{\prime}$ are precisely the points $v_{1}^{\prime}$.

Consider the points $v_{1}$ and the lines $L_{0}$ and $L_{1}$ of $\mathcal{C}$, and the points $v_{0}^{\prime}, v_{1}^{\prime}$ and the lines $L_{0}^{\prime}$ of $\mathcal{D}$. With respect to the $v_{1}$, the $L_{0}$ have span $t_{1}$ and the $L_{1}$ have span $s_{2}$. We place the $v_{0}^{\prime}$ arbitrarily on the lines $L_{0}$, and then we construct the lines $L_{0}^{\prime}$ in $\mathcal{D}$, which are also of span $s_{2}$. By applying Theorem 3 , it follows that the lines $L_{0}^{\prime}$ must intersect the lines $L_{1}$, and the intersection point is precisely the point with label $\left[\left[s_{2}, t_{1}\right]\right]$, namely, $v_{1}^{\prime}$.

Therefore, the combined symbol is admissible.

\subsection{Using trivial three-ring celestial configurations}

One very easy class of movable configurations to construct is based on trivial threering celestial configurations $m \#(a, b ; c, a ; b, c)$. Using Theorem 7 , the movable configuration

$$
m \#(a, * b ; c, a ; b, c) \$(* c, b ; a, c ; b, a)
$$

may be constructed whenever $b$ and $c$ are both odd; note that $m \#(a, b ; c, a ; b, c)$ and $m \#(c, b ; a, c ; b, a)$ represent the same geometric configuration, but the labelling is reversed. The smallest such example is given in Figure 7.

\subsection{Using two astral $\left(n_{4}\right)$ configurations}

In [5], astral $\left(n_{4}\right)$ configurations - that is, $\left(n_{4}\right)$ configurations with precisely two symmetry classes each of points and lines - were completely characterized. Some astral $\left(n_{4}\right)$ configurations, called type 1 in [2,3,4], are celestial configurations. An important result from [5], originally conjectured by Branko Grünbaum in [10], was to prove the following (modified slightly to use the current notation for celestial configurations):

Theorem 8. All celestial astral $\left(n_{4}\right)$ configurations are listed in the following: there are two infinite families, Family, 1: $(6 k) \#(3 k-j,|3 k-2 j|, j, 2 k)$ for $j=1, \ldots, 2 k-$ $1, j \neq k$ and $j \neq \frac{3 k}{2}$; and Family $2:(6 k) \#(3 k-2 j, j, 2 k, 3 k-j)$, for $j=1, \ldots, k-1$, for $k=2,3,4, \ldots$ There are 27 sporadic configurations, with $m=30,42$, and 60 , where a configuration is sporadic if it is not a member of one of the infinite families. Finally, there are multiples of the sporadic configurations. 
Table 2: The sporadic celestial astral $\left(n_{4}\right)$ configurations.

$$
\begin{aligned}
& m=30 \\
& 30 \#(4,1 ; 6,7) \quad 30 \#(6,1 ; 4,7) \quad 30 \#(6,1 ; 10,11) \\
& 30 \#(6,2 ; 6,8) \quad 30 \#(7,2 ; 11,12) \quad 30 \#(8,1 ; 12,13) \\
& 30 \#(10,1 ; 6,11) \quad 30 \#(10,6 ; 10,12) \quad 30 \#(10,7 ; 12,13) \\
& 30 \#(11,2 ; 7,12) \quad 30 \#(11,6 ; 13,14) \quad 30 \#(12,1 ; 8,13) \\
& 30 \#(12,4 ; 12,14) \quad 30 \#(12,7 ; 10,13) \quad 30 \#(13,6 ; 11,14) \\
& m=42 \\
& 42 \#(6,1 ; 12,13) \quad 42 \#(11,6 ; 17,18) \quad 42 \#(12,1 ; 6,13) \\
& 42 \#(12,5 ; 18,19) \quad 42 \#(17,6 ; 11,18) \quad 42 \#(18,5 ; 12,19) \\
& m=60 \\
& 60 \#(9,2 ; 21,22) \quad 60 \#(12,5 ; 24,25) \quad 60 \#(14,3 ; 26,27) \\
& 60 \#(21,2 ; 9,22) \quad 60 \#(24,5 ; 12,25) \quad 60 \#(26,3 ; 14,27)
\end{aligned}
$$

The sporadic configurations with $m=30,42$, and 60 are listed in Table 2. A necessary condition for an astral celestial $\left(n_{4}\right)$ configuration to exist is that $n=$ $12 k$, for some natural number $k$. Here, a multiple refers to taking some number of concentric copies of a configuration, rotated so that they are equally spaced. Note that in the notation of $[3,4,5,9,10]$, a celestial astral $\left(n_{4}\right)$ configuration was denoted as $m \# a_{b} c_{d}$, which corresponds to the configuration $m \#(a, b ; d, c)$ in the notation of this paper. Finally, a remark: in the first infinite family, since $j$ can be as large as $2 k-1$, it is possible for the quantity $3 k-2 j$ to become negative, hence the need for the absolute value. The quantity $3 k-2 j$ in family 2 must always be positive, as $j<k$ in that family.

Of particular interest in the construction of movable configurations are the two infinite families, since inspection shows that none of the sporadic configurations (or any multiples of those) is point- or line-modifiable.

In Family 1, $(6 k) \#(3 k-j, 3 k-2 j ; j, 2 k)$, by Lemma 1 , half the lines in the symmetry class $L_{0}$ may be removed when $3 k-j$ and $3 k-2 j$ are both odd, which happens when $j$ is even and $k$ is odd. In Family 2, $(6 k) \#(3 k-2 j, j ; 2 k, 3 k-j)$, half the lines in symmetry class $L_{0}$ may be removed when $3 k-2 j$ and $j$ are both odd, which happens when $j$ and $k$ are both odd. (Since $2 k$ is always even, the lines 
labelled $L_{1}$ can never have half of them removed.) Similarly, using Lemma 2, half the points labelled $v_{1}$ of a configuration in Family 1 may be removed when $j$ and $k$ are both odd, while in Family 2 half the points labelled $v_{0}$ may be removed when $k$ is odd and $j$ is even. In particular, we will not be able to construct movable configurations in the case that $k$ is even!

Notice that if $k$ is odd, $\frac{3 k}{2}$ is never an integer, so there are $2(k-1)$ configurations in Family 1 (since $j \neq k$ ) and $k-1$ configurations in Family 2. Each configuration, modified appropriately, may participate as half of a movable configuration. In order to determine important characteristics of these movable configurations, we need to carefully investigate the relationship between members of the infinite families; in particular, it is helpful to determine which configurations are polars of which others.

In [8], it is shown that the polar of a celestial configuration $m \#\left(s_{1}, t_{1} ; \ldots ; s_{h}, t_{h}\right)$ may be obtained by permuting the sequence $\left(s_{1}, t_{1} ; \ldots ; s_{h}, t_{h}\right)$ an odd number of steps (and by reversals of such permutations). We can use this to analyze the polarity relationships among the astral celestial $\left(n_{4}\right)$ configurations which are members of the inifinte families.

Theorem 9. The polar of the configuration $6 k \#(3 k-2 j, j ; 2 k, 3 k-j)$ in Family 2 is the configuration $6 k \#(3 k-j, 3 k-2 j ; j, 2 k)$ in Family 1 , where $j<k$. The polar of the configuration $6 k \#(3 k-j, 3 k-2 j ; j, 2 k)$ in Family 1 where $j>k$ is the configuration $6 k \#(j, 3 k-2 j ; 3 k-j, 2 k)$, also in Family 1 with $j>k$.

Proof. In Family 2, where $j=1,2, \ldots, k-1$, cyclically permuting the sequence $(3 k-2 j, j ; 2 k, 3 k-j)$ one step forward yields $(3 k-j, 3 k-2 j ; j, 2 k)$, and $6 k \#(3 k-$ $j, 3 k-2 j ; j, 2 k)$ is a member of Family 1 , with $j<k$.

This leaves the other $k-1$ members of Family 1 unaccounted for, where $j=$ $k+1, k+2, \ldots, k+(k-1)=2 k-1$. Let $j=k+q$, and suppose $q<\frac{k}{2}$; then

$$
\begin{aligned}
6 k \#(3 k-j,|3 k-2 j| ; j, 2 k) & =6 k \#(2 k-q,|k-2 q|, k+q, 2 k) \\
& =6 k \#(2 k, k+q ; k-2 q, 2 k-q) .
\end{aligned}
$$

On the other hand, if $j=2 k-q$ and $q<\frac{k}{2}$, then

$$
\begin{aligned}
6 k \#(3 k-j,|3 k-2 j| ; j, 2 k) & =6 k \#(k+q,|-(k-2 q)|, 2 k-q, 2 k) \\
& =6 k \#(k+q, k-2 q, 2 k-q, 2 k),
\end{aligned}
$$

which is clearly the polar of $6 k \#(2 k, k+q ; k-2 q, 2 k-q)$, since the sequence is cyclically shifted by a single step. Unsubstituting $j=k+q$, we see that the polar of $6 k \#(3 k-j,|3 k-2 j| ; j, 2 k)$ is $6 k \#(j,|3 k-2 j| ; 3 k-j, 2 k)$. 
We can use Theorem 7 and the results on polar pairs of astral configurations to completely determine all movable configurations formed using two astral celestial configurations.

Theorem 10. The only movable $\left(n_{4}\right)$ configurations obtained from two astral celestial configurations are the following:

1. $6 k \#(2 k, * 3 k-j ; 3 k-2 j, j) \$(* 3 k-j, 3 k-2 j ; j, 2 k)$, for $j$ even, $k$ odd, and $j \leq k-1$;

2. $6 k \#(3 k-j, * 3 k-2 j ; j, 2 k) \$(* 3 k-2 j, j ; 2 k, 3 k-j)$, for $j$ and $k$ both odd, and $j \leq k-1$;

3. $6 k \#(3 k-j, * 3 k-2 j ; j, 2 k) \$(* j, 3 k-2 j ; 3 k-j, 2 k)$, for $j$ and $k$ odd and $j \geq k+1$;

4. $6 k \#(j, * 3 k-2 j ; 3 k-j, 2 k) \$(* 3 k-j, 3 k-2 j ; j, 2 k)$, for $j$ even, $k$ odd, and $j \geq k+1$;

where $k$ is at least 3. Movable configurations obtained in this way are, in general, distinct. In addition, the two factors form a polar pair of astral celestial configurations, and every polar pair of astral celestial configurations which are not sporadic gives rise to a movable configuration.

Proof. We will analyze several cases, corresponding to when astral configurations are point- or line-modifiable, and given a point-modifiable configuration, we will determine another factor that is line-modifiable and which produces an admissible combined symbol. Note that since no sporadic configurations are point- or linemodifiable, it is sufficient to consider only the infinite families of astral configurations.

Case 1: $j$ is even, $k$ is odd and $j \leq k-1$. In this case, the Family 2 configuration

$$
6 k \#(3 k-2 j, j ; 2 k, 3 k-j)=6 k \#(2 k, 3 k-j ; 3 k-2 j, j)
$$

is point-modifiable into $6 k \#(2 k, * 3 k-j ; 3 k-2 j, j)$, since $3 k-j$ and $3 k-2 j$ are both odd. Theorem 7 says that a configuration whose symbol is of the form $(3 k-2 j, 3 k-j ;-,-)$ will be a compatible line-modifiable configuration. Using Theorem 9 , the polar to the configuration, namely

$$
\begin{aligned}
6 k \#(3 k-j, 3 k-2 j ; j, 2 k) & =6 k \#(j, 2 k ; 3 k-j, 3 k-2 j) \\
& =6 k \#(3 k-2 j, 3 k-j ; 2 k, j)
\end{aligned}
$$

THE ELECTronic JOURNAL of COMBinatorics 13 (2006), \#R104 
has such a beginning and is a compatible line-modifiable configuration. Thus, every configuration in Family 2 where $j$ is even, $k$ is odd, and $j<k$ participates in the movable configuration

$$
6 k \#(2 k, * 3 k-j ; 3 k-2 j, j) \$(* 3 k-2 j, 3 k-j ; 2 k, j) .
$$

By construction, the component configurations form a polar pair.

These configurations account for half of the configurations from Family 2, and one quarter of the configurations from Family 1. The smallest such configuration, $18 \#(6, * 7 ; 5,2) \$(* 7,5 ; 2,6)$, is shown in Figure 9 .

Case 2: $j$ and $k$ are both odd, and $j \leq k-1$. If $j$ and $k$ are both odd, and $j<$ $k$, then configurations in Family 1 are point-modifiable, since $3 k-2 j$ and $j$ are both odd. Theorem 7 says that we need to find a configuration that is compatible with $6 k \#(3 k-j, * 3 k-2 j ; j, 2 k)$; that is, we need a configuration with sequence of the form $(j, 3 k-2 j ;-,-)$. The polar of the configuration, a member of Family 2, again will work, since

$$
\begin{aligned}
6 k \#(3 k-2 j, j ; 2 k, 3 k-j) & =6 k \#(2 k, 3 k-j ; 3 k-2 j, j) \\
& =6 k \#(j, 3 k-2 j ; 3 k-j, 2 k) .
\end{aligned}
$$

That is, all configurations in Family 1 and 2 with $j$ and $k$ odd and $j<k$ participate in a movable configuration of the form

$$
6 k \#(3 k-j, * 3 k-2 j ; j, 2 k) \$(* j, 3 k-2 j ; 3 k-j, 2 k) .
$$

Note that these configurations account for the other half of the configurations from Family 2 and another quarter of the configurations from Family 1. The smallest such configuration, $18 \#(8, * 7 ; 1,6) \$(* 1,7 ; 8,6)$, is shown in Figure 10 .

Case 3: $k$ is odd, $j$ is odd and $j \geq k+1$. If $j$ is odd, then $3 k-2 j$ and $j$ are both odd. If we consider the configuration in Family 1 with symbol $6 k \#(3 k-$ $j, 3 k-2 j ; j, 2 k)$, which is point-modifiable, Theorem 7 says that a compatible configuration will have a sequence of the form $(j, 3 k-2 j ;-,-)$. The polar configuration $m \#(j, 3 k-2 j ; 3 k-j, 2 k)$ works nicely. That is, if $j$ is odd, we can construct the movable configuration

$$
6 k \#(3 k-j, * 3 k-2 j ; j, 2 k) \$(* j, 3 k-2 j ; 3 k-j, 2 k) .
$$

The smallest such configuration, $18 \#(4, * 1 ; 5,6) \$(* 5,1 ; 4,6)$, is shown in Figure 11. 


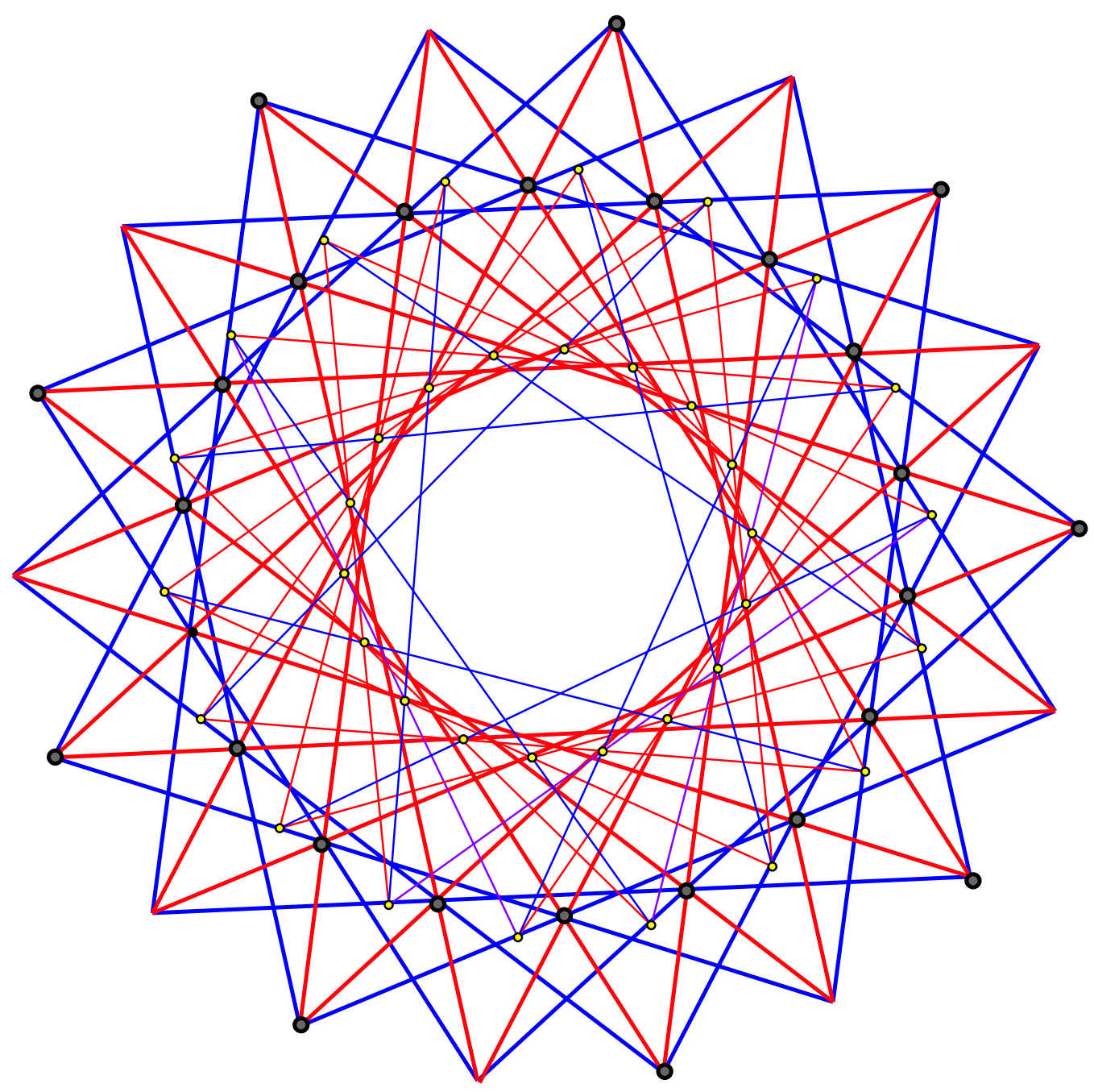

Figure 9: $18 \#(6, * 7 ; 5,2) \$(* 7,5 ; 2,6)$, the smallest movable configuration constructed from two astral $\left(n_{4}\right)$ configurations satisfying the situation of Case 1 in the proof of Theorem 10. 


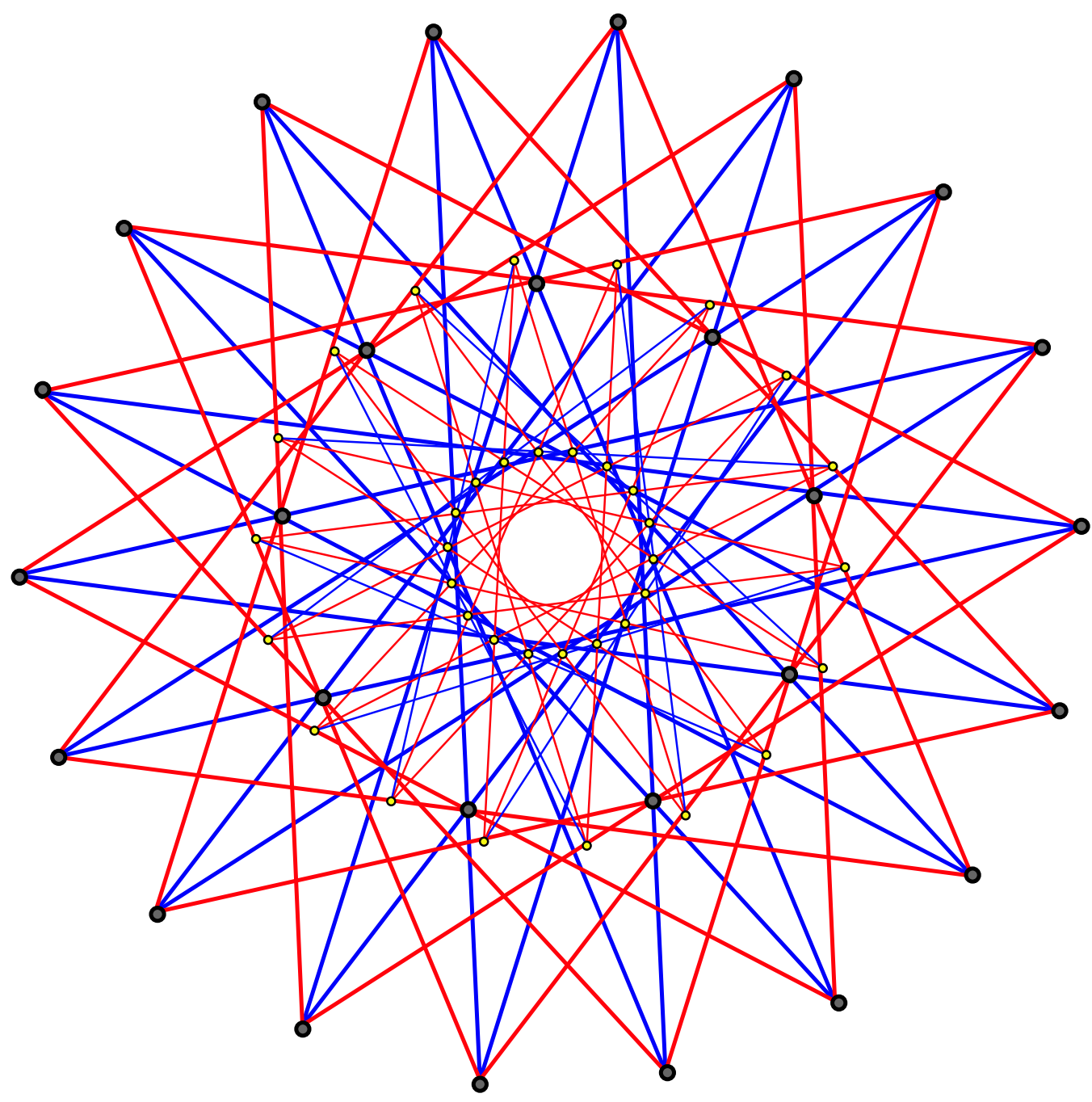

Figure 10: $18 \#(8, * 7,1,6) \$(* 7,1,6,8)$, the smallest movable configuration constructed from two astral $\left(n_{4}\right)$ configurations satisfying the situation of Case 2 in the proof of Theorem 10. 


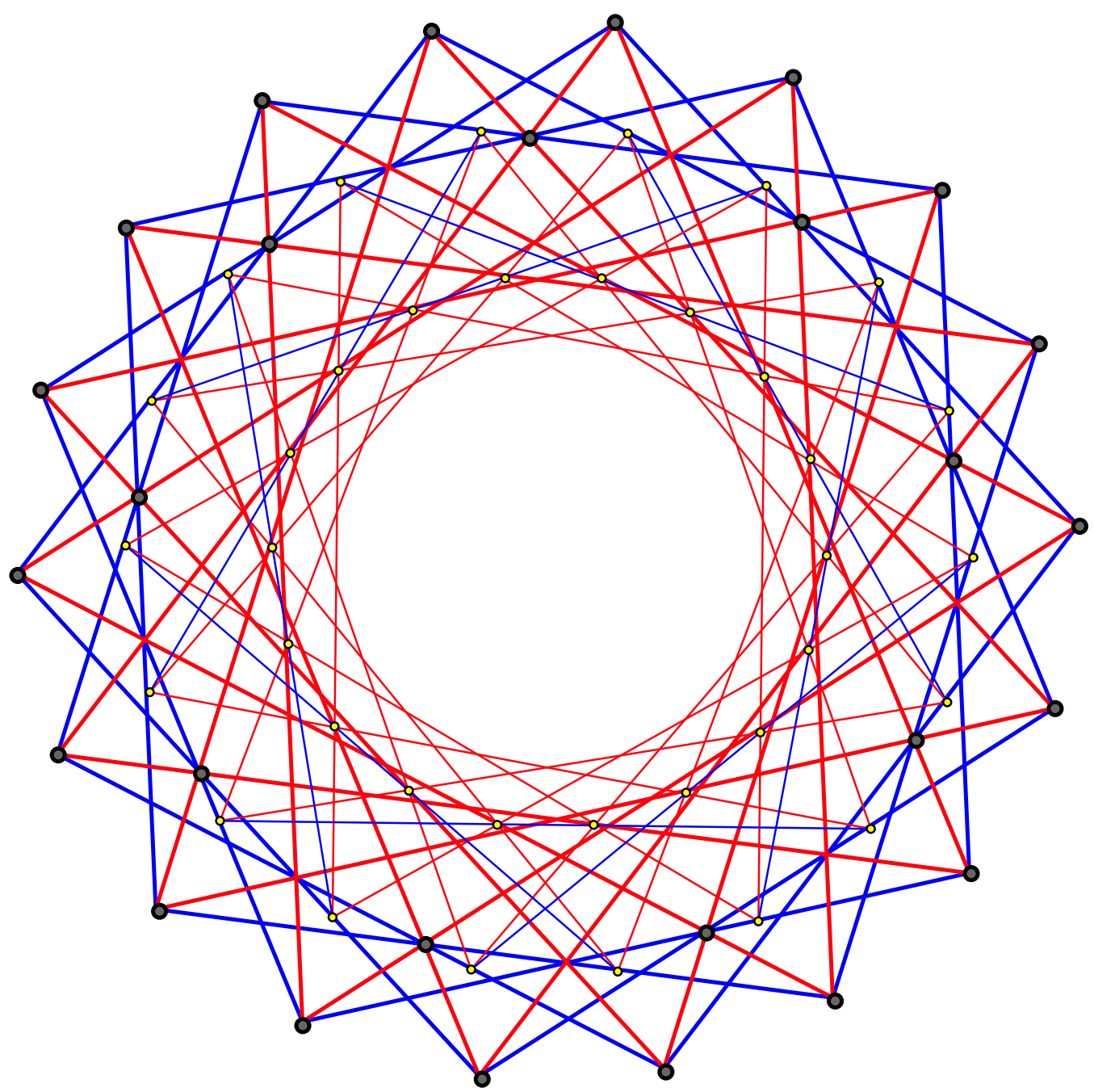

Figure 11: $18 \#(4, * 1 ; 5,6) \$(* 5,1 ; 4,6)$, the smallest movable configuration constructed from two astral $\left(n_{4}\right)$ configurations satisfying the situation of Case 3 in the proof of Theorem 10 
Case 4: $k$ is odd, $j$ is even, and $j>k$. If $j$ is even, $6 k \#(3 k-j, 3 k-2 j ; j, 2 k)$ is not point-modifiable; however, it is line-modifiable, since $3 k-j$ and $3 k-$ $2 j$ are both odd. Again applying Theorem 7, we see that the configuration $6 k \#(j, 3 k-2 j ; 3 k-j, 2 k)$, which is now point-modifiable, will be compatible, so if $j$ is even, we can construct the movable configuration

$$
6 k \#(j, * 3 k-2 j ; 3 k-j, 2 k) \$(* 3 k-j, 3 k-2 j ; j, 2 k) .
$$

Cases 3 and 4 account for the rest of the configurations in Family 1, those with $j>k$.

Note that if $k=3$, there are only two configurations where $j>k$, so Case 3 and Case 4 produce the same movable configuration. The smallest movable configurations which are different in Case 3 and 4 are

$$
30 \#(8, * 1 ; 7,10) \$(* 7,1 ; 8,10) \quad \text { (in case } 3)
$$

and

$$
30 \#(6, * 3 ; 9,10) \$(* 9,3 ; 6,10) \quad \text { (in case } 4) .
$$

It follows that all astral $\left(n_{4}\right)$ configurations in the infinite families participate in some movable configuration, and the configurations produced are distinct, as long as $k$ is larger than 3. By construction, the two factors in each movable configuration form a polar pair.

\section{More complicated movable configurations}

It is possible to construct movable configurations with multiple degrees of freedom. To do this, we will need to generalize the notion of admissible combined symbols: we call a combined symbol

$$
m \#\left(s_{1}, t_{1} ; \ldots ; s_{i}, * t_{i} ; \ldots ; s_{h}, t_{h}\right) \$\left(* q_{1}, r_{1} ; \ldots ; q_{h}, r_{h}\right)
$$

admissible if when the points $v_{0}^{\prime}$ of the second modified configuration $m \#\left(* q_{1}, r_{1} ; \ldots ; q_{k}, r_{k}\right)$ are placed on the lines $L_{i-1}$ of the first modified configuration $m \#\left(s_{1}, t_{1} ; \ldots ; s_{i}, * t_{i} ; \ldots ; s_{h}, t_{h}\right)$, then the points $v_{1}^{\prime}$ of the second modified configuration lie on the lines $L_{i}$ of the first modified configuration. These generalized admissible combined configurations again correspond to $\left(n_{4}\right)$ configuration; the proof is a straightforward generalization of the proof of Theorem 6 . 


\section{$5.1 \quad$ Multiply-nested configurations}

Note that in

$$
m \#(a, * b ; c, a ; b, c) \$(* c, b ; a, c ; b, a),
$$

through the points labelled $v_{0}^{\prime}$ we have lines labelled $L_{0}^{\prime}$ of span $c$ (of which half have been removed) and $a$ (with label $L_{1}^{\prime}$ ).

Consider the sequence $(c, b ; a, * c ; b, a)$. This indicates that we should place new points $v_{0}^{\prime \prime}$ on the lines $L_{1}^{\prime}$ of span $a$ (with respect to the points $v_{2}^{\prime}$ ) and delete half the points with symbol $v_{2}^{\prime}$. According to Lemma 2, this is possible since $c$ and $b$ are both odd. Using Theorem 7 , the modified configuration $m \#(c, b ; a, * c ; b, a)$ is compatible with the configuration $m \#(* b, c ; a, b ; c, a)$. The combination of these two configurations does not involve the lines $L_{0}^{\prime}$, half of which have been deleted, so in fact, the configuration $m \#(* b, c ; a, b ; c, a)$ is compatible with the movable configuration $m \#(a, * b ; c, a ; b, c) \$(* c, b ; a, c ; b, a)$ ! That is, we can construct the admissible extended combined symbol

$$
m \#(a, * b ; c, a ; b, c) \$(* c, b ; a, * c ; b, a) \$(* b, c ; a, b ; c, a)
$$

which has two degrees of freedom. An example of such a configuration,

$$
8 \#(2, * 1 ; 3,2 ; 1,3) \$(* 3,1 ; 2, * 3 ; 1,2) \$(* 1,3 ; 2,1 ; 3,2)
$$

is given in Figure 12.

These configurations may be nested as deeply as one wishes: The extended combined symbol in general is of the form

$$
\begin{gathered}
m \#\left(s_{1,1}, * t_{1,1} ; \ldots ; s_{1, h_{1}}, t_{1, h_{1}}\right) \\
\ldots\left(* s_{2,1}, t_{2,1} ; \ldots ; s_{2, j_{1}}, * t_{2, j_{1}} ; \ldots ; s_{2, h_{2}}, t_{2, h_{2}}\right) \\
\ldots\left(* s_{n-1,1}, t_{n-1,1} ; \ldots ; s_{i, j_{n-1}}, * t_{i, j_{n-1}} ; \ldots ; s_{n-1, h_{n-1}}, t_{n-1, h_{n-1}}\right) \\
\quad \$\left(* s_{n, 1}, t_{n, 1} ; \ldots ; s_{n, h_{n}}, t_{n, h_{n}}\right)
\end{gathered}
$$

where

$$
\left(* s_{i, 1}, t_{i, 1} ; \ldots ; s_{i, j_{i}}, * t_{i, j_{i}} ; \ldots ; s_{i, h_{i}}, t_{i, h_{i}}\right),
$$

indicates that the configuration $m \#\left(* s_{i, 1}, t_{i, 1} ; \ldots ; s_{i, j_{i}}, * t_{i, j_{i}} ; \ldots ; s_{i, h_{i}}, t_{i, h_{i}}\right)$ has been modified by removing half the lines labelled $L_{0}$ and half the points labelled $\left[\left[s_{i, j_{i}}, t_{i, j_{i}}\right]\right]$. Each consecutive pair of configuration sequences must correspond to compatible configurations. 


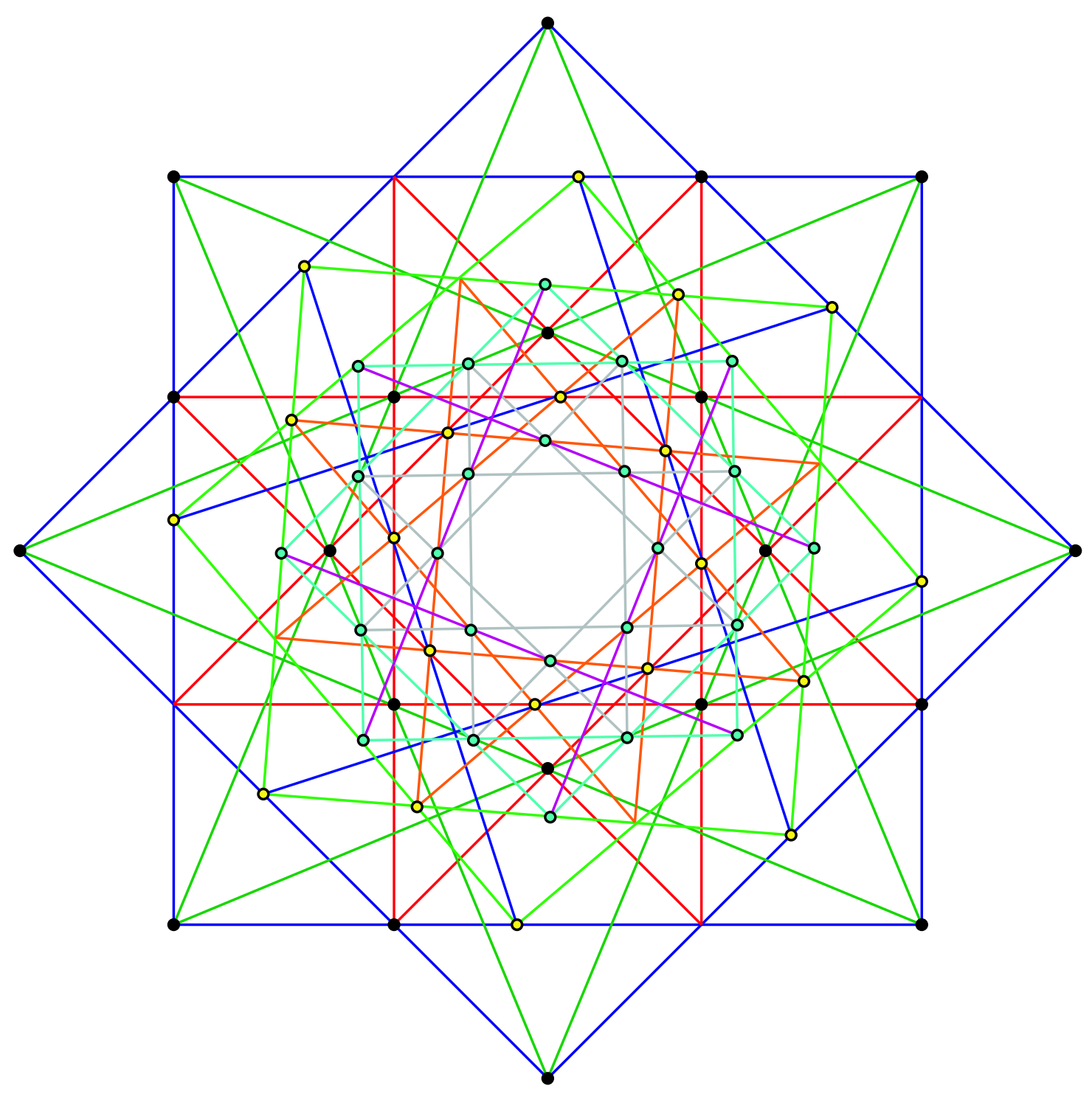

Figure 12: A $\left(144_{4}\right)$ movable configuration

$$
8 \#(2, * 1 ; 3,2 ; 1,3) \$(* 3,1 ; 2, * 3 ; 1,2) \$(* 1,3 ; 2,1 ; 3,2),
$$

with two degrees of freedom. Each of the component modified configurations is shown with different colored vertices (black, yellow, and blue). 


\subsection{Multiple configurations nested on a single configuration}

As long as the entries are far enough apart, so that new vertices $v_{0}^{\prime}$ and $v_{0}^{\prime \prime}$ are placed on different lines and the new intersections appear on different lines, it is possible to nest multiple celestial configurations on a single initial configuration.

As an explicit example, consider the trivial four-ring celestial configuration $m \#(a, b ; c, d ; b, a ; d, c)$, where $a, b, c$ and $d$ are all distinct.

Using the instructions in Theorem 7 , we can straightforwardly construct several movable configurations from this original configuration: if $b$ and $c$ are odd,

$$
m \#(a, * b ; c, d ; b, a ; d, c) \$(* c, b ; a, d ; b, c ; d, a)
$$

is admissible, while if $a$ and $d$ are odd,

$$
m \#(a, b ; c, d ; b, * a ; d, c) \$(* d, a ; b, c ; a, d ; c, b),
$$

is admissible, among others. The first movable configuration has the points $v_{0}^{\prime}$ placed on the lines $L_{0}$ with new intersections on the lines $L_{1}$; the second movable configuration has the points $v_{0}^{\prime \prime}$ placed on the lines $L_{2}$ with new intersections on the lines $L_{3}$.

Therefore, we can construct a movable configuration using both these new configurations by beginning with the modified configuration $m \#(a, * b ; c, d ; b, * a ; d, c)$, which has half the points $v_{1}$ of label $[[a, b]]$ and $v_{3}$ of label $[[b, a]]$ removed, and simultaneously placing new points $v_{0}^{\prime}$ on lines $L_{0}$ and $v_{0}^{\prime \prime}$ on lines $L_{2}$. These new points are then used to construct the configurations $m \#(* c, b ; a, d ; b, c ; d, a)$ beginning with the $v_{0}^{\prime}$ and $m \#(* d, a ; b, c ; a, d ; c, b)$ beginning with the $v_{0}^{\prime \prime}$. Half the points with label $v_{1}$ and $v_{3}$ are removed, so each line $L_{i}$ has one point $v_{j}$ removed for some choice of $j$, and each line $L_{i}$ has one new point, either $v_{j}^{\prime}$ or $v_{j}^{\prime \prime}$, again, for an appropriate value of $j$, added.

Notation for such a configuration is

$$
m \#\left(s_{1}, * t_{1} ; \ldots ; s_{i_{1}}, * t_{i_{1}} ; \ldots ; s_{i_{q}}, * t_{i_{q}} ; \ldots ; s_{h}, t_{h}\right) \$\left\{\begin{array}{c}
\left(* s_{1,1}, t_{1,1} ; \ldots ; s_{1, h_{1}}, t_{1, h_{1}}\right) \\
\vdots \\
\left(* s_{q, 1}, t_{q, 1}, \ldots, s_{q, h_{q}}, t_{q, h_{q}}\right)
\end{array}\right.
$$

where the $j$-th modified configuration after the brace, with sequence $\left(* s_{j 1}, t_{j 1} ; \ldots ; s_{j h_{j}}, t_{j h_{j}}\right)$, is to be constructed using points $v_{0}^{(j)}$ placed on lines $L_{i-1}$ of the configuration before the brace, indicated by $* t_{i_{j}}$ in that configuration. Clearly, 
arbitrarily many configurations may be nested in this fashion if the original configuration has enough rings ( $h$ is large enough in the configuration before the brace); $k$ configurations may be nested if $h$ is at least $4 k$ (and the parities of the $s_{i}$ and $t_{i}$ are appropriate in all the configurations).

For example, the smallest of these configurations has $m=16$ (since we need $a$, $b, c$, and $d$ to be odd). One such configuration is

$$
16 \#(7, * 5 ; 3,1 ; 5, * 7 ; 1,3) \$\left\{\begin{array}{l}
(* 3,5 ; 7,1 ; 5,3 ; 1,7) \\
(* 1,7 ; 5,3 ; 7,1 ; 3,5)
\end{array}\right.
$$

unfortunately, the resulting configuration is too complicated to display intelligibly in this paper.

\section{Some open questions}

By a symmetric configuration I mean a configuration with non-trivial geometric symmetry. The discovery of highly symmetric $\left(n_{4}\right)$ configurations with non-trivial degrees of freedom was quite unexpected; it highlights the fact that general $\left(n_{4}\right)$ configurations - even those with relatively high degrees of symmery, as these movable configurations have - are much stranger than might have been anticipated when celestial configurations and astral configurations were the only infinite classes of highly symmetric $\left(n_{4}\right)$ configurations that were well understood.

Question 1. Are there symmetric $\left(n_{4}\right)$ configurations with a continuous parameter where the number of points in a single symmetry class is odd?

Question 2. Is there some $N$ so that for all $n \geq N$, there exists a movable $\left(n_{4}\right)$ configuration (symmetric or not)?

There exist highly symmetric (in fact, astral) classes of movable $\left(n_{3}\right)$ configurations.

Question 3. Does there exist a symmetric, movable $\left(p_{q}, n_{k}\right)$ configuration, where one of $q$ and $k$ is at least 4? If so, are there infinite families of such configurations?

Interactive examples of the movable configurations shown in this paper are available from the author's website:

http://webpages . ursinus.edu/lberman/MovableConfigurations.html .

The Electronic Journal of Combinatorics 13 (2006), \#R104 


\section{Acknowledgments}

The author wishes to thank Branko Grünbaum and the anonymous referee for extremely helpful comments; Ilana Williams also contributed in her own unique way.

\section{References}

[1] Altschiller-Court, Nathan. College Geometry. (1952) Barnes \& Noble, Inc.

[2] Berman, L.W. Some results on odd astral configurations. The Electronic Journal of Combinatorics 13 (2006) \#R27.

[3] Berman, L.W. Even astral configurations. The Electronic Journal of Combinatorics 11 (2004) \#R37

[4] Berman, L.W. Astral Configurations. Ph.D. Thesis, University of Washington. (2002)

[5] Berman, L.W. A characterization of astral $\left(n_{4}\right)$ configurations. Discrete and Computational Geometry. 26 (2001) no. 4, 603 - 612.

[6] Boben, M. and T. Pisanski. Polycyclic configurations. European Journal of Combinatorics. 244 (2003), 431 - 457.

[7] Bokowski, J. and L. Schewe. There are no realizable $15_{4^{-}}$and $164^{-}$ configurations. Rev. Roumaine Math. Pures Appl. 50 (2005) no. 5-6, $483-$ 493.

[8] Grünbaum, B. Configurations of points and lines. In The Coxeter Legacy: Reflections and Projections., Chandler Davis and Erich W. Ellers, eds. American Mathematical Society, (2006), 179 - 225.

[9] Grünbaum, B. Configurations. Unpublished manuscript/class notes. Spring 2004.

[10] Grünbaum, B. Astral $\left(n_{4}\right)$ configurations. Geombinatorics 9 (2000), $127-134$.

[11] Grünbaum, B. Which $\left(n_{4}\right)$ configurations exist? Geombinatorics 9 (2000), 164 $-169$.

[12] Grünbaum, B. Configurations. Unpublished manuscript/class notes. 1991.

[13] Grünbaum, B. and J. F. Rigby. The real configuration $\left(21_{4}\right)$. J. London Math. Soc (2). 41 (1990), $336-346$.

[14] Marušič, D. and T. Pisanski, Weakly flag-transitive configurations and half-arc transitive graphs. European J. Combin. 20 (1999), no. 6, 559 - 570. 